\title{
Interfacing the DEC LPS 11 to electromechanical laboratory apparatus
}

\author{
W. LLOYD MILLIGAN and WILLIAM NEWS \\ Veterans Administration Hospital, Columbia, South Carolina 29201
}

\begin{abstract}
A hardware-software extension to the DEC LPS 11 facilitates interfacing the PDP 11 to external instruments. The hardware component consists of eight relay drivers and relays, four contact conditioners, and an interrupt pulse former. FORTRAN-callable RT-11 MACRO subroutines control the device, service interrupts, and transfer control to FORTRAN completion routines.
\end{abstract}

The LPS 11 is a real-time subsystem of the Digital Equipment Corporation (DEC) PDP 11 computer. This system includes a programmable real-time clock, eight-channel multiplexer, analog-digital (A-D) converter, and optional accessories. The LPSDR-A option contains two 16-bit registers for TTL input/output $(\mathrm{I} / 0)$. The present paper describes a device which utilizes these registers and the related status register to interface the PDP 11 computer to conventional (transistor, vacuum tube, and electromechanical) laboratory apparatus. This device, the Digital I/O Expander (DIOX), is operated under program control at the RT-11 FORTRAN level by CALLs to assembly language subroutines.

The DIOX consists of two power supplies $(5 \mathrm{~V} \mathrm{dc}$ and $20 \mathrm{~V} \mathrm{dc}$ ), four set-reset type contact conditioners (CXs) whose outputs are connected to Bits $0-3$ of the LPSDR input register, eight relay drivers whose inputs are connected to Bits $0-7$ of the LPSDR output register, and one 100-nsec pulse former whose output is connected to the "external new data ready" (interrupt) input. Relays and indicator LEDs are self-contained in the DIOX. Relay contacts are connected to NuWay studs mounted on a Plexiglas front panel. Standard threeconductor phone jacks are used to convey external inputs to the CXs and pulse former.

\section{CIRCUIT DESCRIPTION}

Figures 1A, 1B, and 1C show the three types of circuits which comprise the DIOX. Circuit $1 \mathrm{~A}$ is a commonly used SPDT contact debouncer (see Lancaster, 1976). Four of these are connected to the four lowest bits of the LPSDR input register. The power supply and 25-pin connector grounds are made common. Figure 1B shows the relay driver and indicator circuit. Eight of these are connected to the low-order byte of the output register. If the 7407 hex buffer is used, the open-circuit voltage should not exceed $30 \mathrm{~V}$ and the closed-circuit current should not exceed $30 \mathrm{~mA}$. Thus, if the operating voltage is $24 \mathrm{~V} \mathrm{dc}$, the relay impedance should be no less than about 800 ohms. Figure 1C shows the interrupt pulse-former circuit which utilizes TTL propogation delays to produce a single negative going pulse of sufficient duration to be detected by the LPSDR interrupt circuit (100 nsec). The pulse-former CX input is connected to a front panel DPDT switch which transfers control between a phone jack, for interfacing external apparatus, and a panel-mounted microswitch for interrupt testing.

\section{SOFTWARE CONTROL}

Software control of the DIOX is accomplished by four FORTRAN-callable assembly language subroutines which function analogously to the RT-11 FORTRAN LPS extensions. These routines (1) close and open the external relays, (2) return to the calling program the value placed in the input register by the external CXs, (3) enable an interrupt to transfer program control to a FORTRAN completion routine selected via the external CXs, and (4) clear the interrupt-enable prior to program termination. The following section describes the function of these routines in detail.

\section{PROGRAMMING THE DIOX FROM RT-11 FORTRAN IV}

\section{EXTREL}

This routine controls the eight external relays in the DIOX. The format of the subroutine call is:

$$
\text { CALL EXTREL( } \mathrm{n}, \mathrm{m})
$$

where $\mathrm{n}$ is an integer expression which specifies a relay number 0 to 7 and $m$ is an integer expression which effects closing (1) or opening (0) the relay. For example,

$$
\text { CALL EXTREL }(5,1)
$$

causes Relay 5 to be closed or

\section{CALL EXTREL $(2,0)$}

causes Relay 2 to be opened. This subroutine has no effect on the LPSDR internal relays, which are con- 

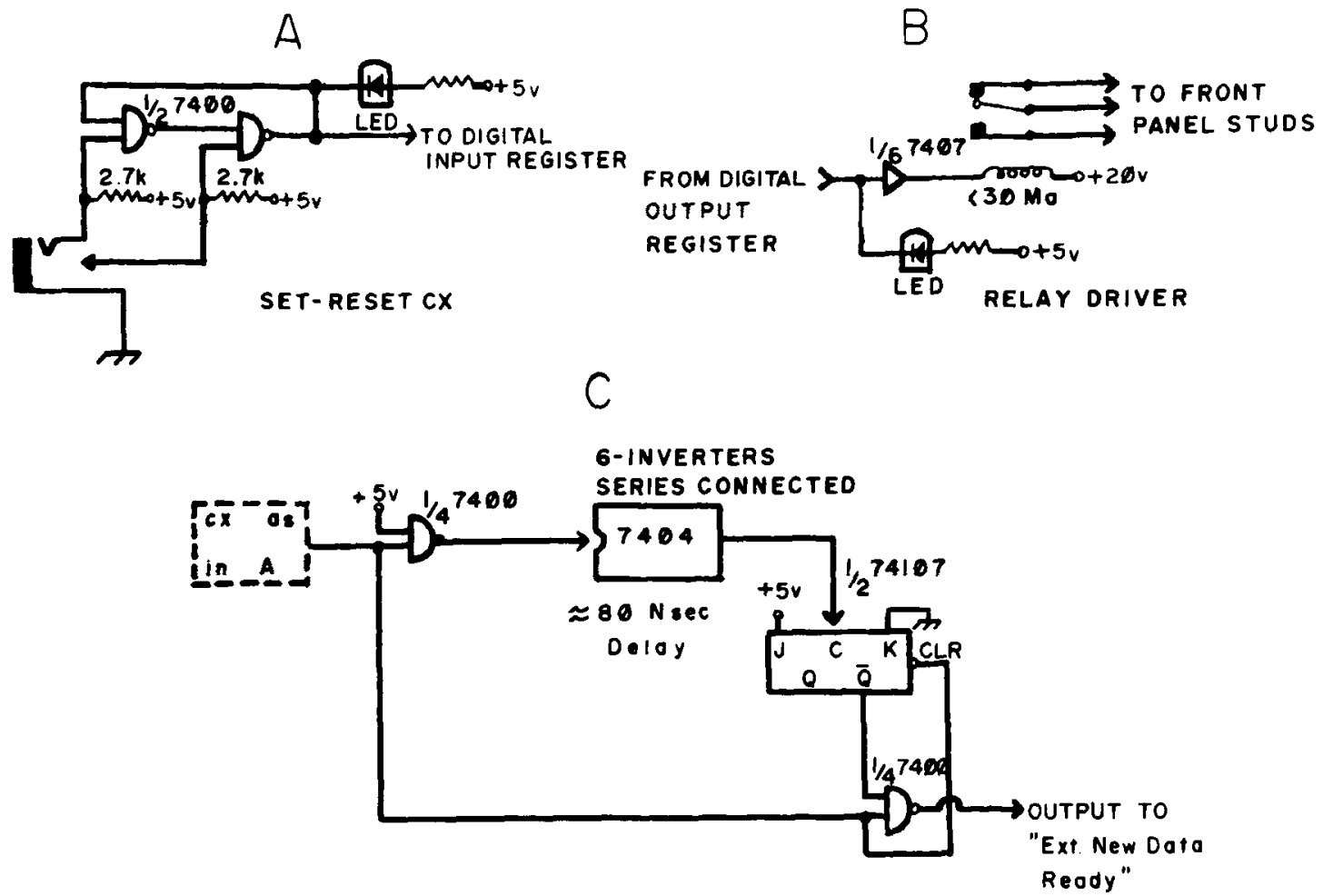

Figure 1. Circuit diagrams showing: (A) set-reset contact conditioner, (B) relay driver-indicator, and (C) interrupt pulse former.

trolled as usual by the CALL REL( $n, m)$ subroutine of the FORTRAN LPS extensions.

\section{READIR}

This subroutine returns the value in the LPSDR input register. The input register and the input bit of the status register are then cleared. The format of the subroutine call is:

\section{CALL READIR(nvar)}

where nvar denotes the integer variable which will contain the value from the input register upon return. The DIOX contains four contact conditioners (CXs) that are connected to Bits $0,1,2$, and 3 of the input register. By setting appropriate combinations of CXs, any decimal value from 0 through 15 may be placed in the input register. The FORTRAN program may test this value and make subsequent program execution conditional upon it.

\section{NTRUPT}

This subroutine enables the DIOX to cause an interrupt by sending an "ex.ternal new data ready" pulse to the LPSDR. In the call to NTRUPT, the user transmits a list of FORTRAN completion routine symbolic names. As many as 15 different subroutines may be specified. The value in the external CXs determines the routine to be entered when the interrupt occurs. NTRUPT constructs a map of completion routine addresses and sets up an interrupt service routine which decodes the value in the input register (setting of DIOX CXs) to select the appropriate completion routine. The format of the NTRUPT subroutine call is:

\section{CALL NTRUPT (CMP1,CMP2,,..,CMP15)}

where the CMPi comprise an ordered list of completion routine symbolic names. All arguments to NTRUPT are optional. However, the position of a subroutine name in the argument list determines the input register value (setting of external CXs) which causes it to execute when an interrupt occurs. In the example:

\section{CALL NTRUPT (,CMP2,„CMP5,CMP6)}

CMP2 is entered when the value 2 is set in the CXs prior to the interrupt, CMP5 executes when the value 5 is set, and so on. One example illustrating the use of NTRUPT is the insertion of an intelligent HALT in a real-time experiment. It is not uncommon for an external emergency to require temporary suspension of an experiment. Simply halting program execution without adjusting any session parameters may have untoward effects. However, with the NTRUPT routine, the user can specify a completion routine to be entered, which permits respecification of important parameters prior to resuming the experiment.

\section{CLRINT}

This subroutine clears the interrupt-enable bit in the LPSDR status register so that subsequent pulses on the 
"external new data ready" line have no effect. A call to CLRINT should be placed before exiting a FORTRAN program which calls NTRUPT. Failure to clear the interrupt-enable bit leaves the system vulnerable to an accidental interrupt with no service routine in memory. Such an interrupt typically causes system failure.

A more detailed paper, which includes RT-11 MACRO source listings of the routines EXTREL, READIR, NTRUPT, and CLRINT as well as FORTRAN programming examples, is available from the author.

\section{SUMMARY}

This report has described a device which expands the DEC LPS 11, facilitating its interaction with conventional psychology laboratory apparatus: stimulators, tone oscillators, counters, event recorders, etc. The two internal relays of the LPS 11 can specify, at most, four external states (one of which is usually null). The addition of eight external relays greatly expands this capacity. The four CXs and "external new data ready" pulse former essentially permit externally coded interrupts. RT-11 MACRO assembly language subroutines are used to control the DIOX and service interrupts. These routines may be called from a FORTRAN program which defines the experimental conditions and possibly also acquires data from external instruments. Use of this device and the associated software has resulted in greater flexibility and more harmonious utilization of the real-time computer with other laboratory equipment.

\section{REFERENCE} Lancaster, D. TTL cookbook. Indianapolis: Howard W. Sams,
1976. 